\title{
Application of Lambert $W$ Function to Planck Spectral Radiance Frequencies
}

\author{
Sabaratnasingam Gnanarajan \\ Aruja \& Arjun Pty Ltd., Sydney, Australia \\ Email: rajan.sgnanarajan@gmail.com
}

How to cite this paper: Gnanarajan, S. (2021) Application of Lambert $W$ Function to Planck Spectral Radiance Frequencies. Journal of Applied Mathematics and Physics, 9, 2500-2510.

https://doi.org/10.4236/jamp.2021.910160

Received: September 18, 2021

Accepted: October 24, 2021

Published: October 27, 2021

Copyright $\odot 2021$ by author(s) and Scientific Research Publishing Inc. This work is licensed under the Creative Commons Attribution International License (CC BY 4.0).

http://creativecommons.org/licenses/by/4.0/

\section{(c) (i) Open Access}

\begin{abstract}
Planck's radiation law provides an equation for the intensity of the electromagnetic radiation from a physical body as a function of frequency and temperature. The frequency that corresponds to the maximum intensity is a function of temperature. At a specific temperature, for the frequencies correspond to much less than the maximum intensity, an equation was derived in the form of the Lambert $W$ function. Numerical calculations validate the equation. A new form of solution for the Euler's transcendental equation was derived in the form of the Lambert $W$ function with logarithmic argument. Numerical solutions to the Euler's equation were determined iteratively and iterative convergences were investigated. Numerical coincidences with physical constants were explored.
\end{abstract}

\section{Keywords}

Lambert W Function, Planck Radiation, Euler's Equation, Transcendental Equation, Fine Structure Constant

\section{Introduction}

Lambert $W$ function has applications in science [1] [2] [3] [4], especially in physics [5] [6]. The Lambert $W$ function has applications in quantum statistics, and it is used to derive Wien's displacement law in connection with the Planck's black body spectral distribution [7] [8] [9], but it has not been used to describe the spectral distribution. Here we present an application to determine the frequencies in the Planck's black body spectral distribution, for a specific intensity much less than maximum intensity, at a temperature.

Euler found the solution for the equation $\mathcal{X}^{\mathcal{Y}}=\mathcal{Y}^{\mathcal{X}}$ in the form of the Lambert $W$ function in the $18^{\text {th }}$ century [1]. Recently, an exponential form of this equation was used with iterative technique to find solutions [10] [11], but the it- 
eration progression towards convergence has not been investigated. Here we investigate the iteration progression and found solutions for the Euler's equation for a large range of numbers.

The Lambert $W$ function is defined by $W(\mathcal{X}) \mathrm{e}^{W(\mathcal{X})}=\mathcal{X}$. For real numbers, when $<0, W(\mathcal{X})$ is a double valued function.

In the region $W(\mathcal{X})<-1$, it is denoted as $W_{-1}(\mathcal{X})$, and in the region $W(\mathcal{X})>-1$, it is denoted as $W_{0}(\mathcal{X})$.

The plot $W(\mathcal{X})$ vs $\mathcal{X}$ is shown in Figure 1 , and the plot $\ln (-W(\mathcal{X}))$ vs $\ln (-\mathcal{X})$ for $\mathcal{X}<0$, is shown in Figure 2. Figure 2 displays more detail description of the Lambert $W$ function in the region 0 to $-\infty$.

\section{Lambert W Function and Planck's Radiation Law}

In the Planck's radiation law, the spectral radiance in terms of frequency [7] is given by

$$
B(v, T)=\frac{2 h v^{3}}{c^{2}} \frac{1}{\mathrm{e}^{\frac{h v}{k T}}-1}
$$

The frequency $v_{\max }$ corresponds to the maximum intensity [8] [9] is given by

$$
v_{\max }=\frac{k T}{h}\left[3+W\left(\frac{-3}{\mathrm{e}^{3}}\right)\right]
$$

In the Planck's radiation curve at a temperature, for any one intensity below the maximum intensity, two different frequencies can be found. Consider $v_{1}$ and $v_{2}$ are two frequencies correspond to one intensity.

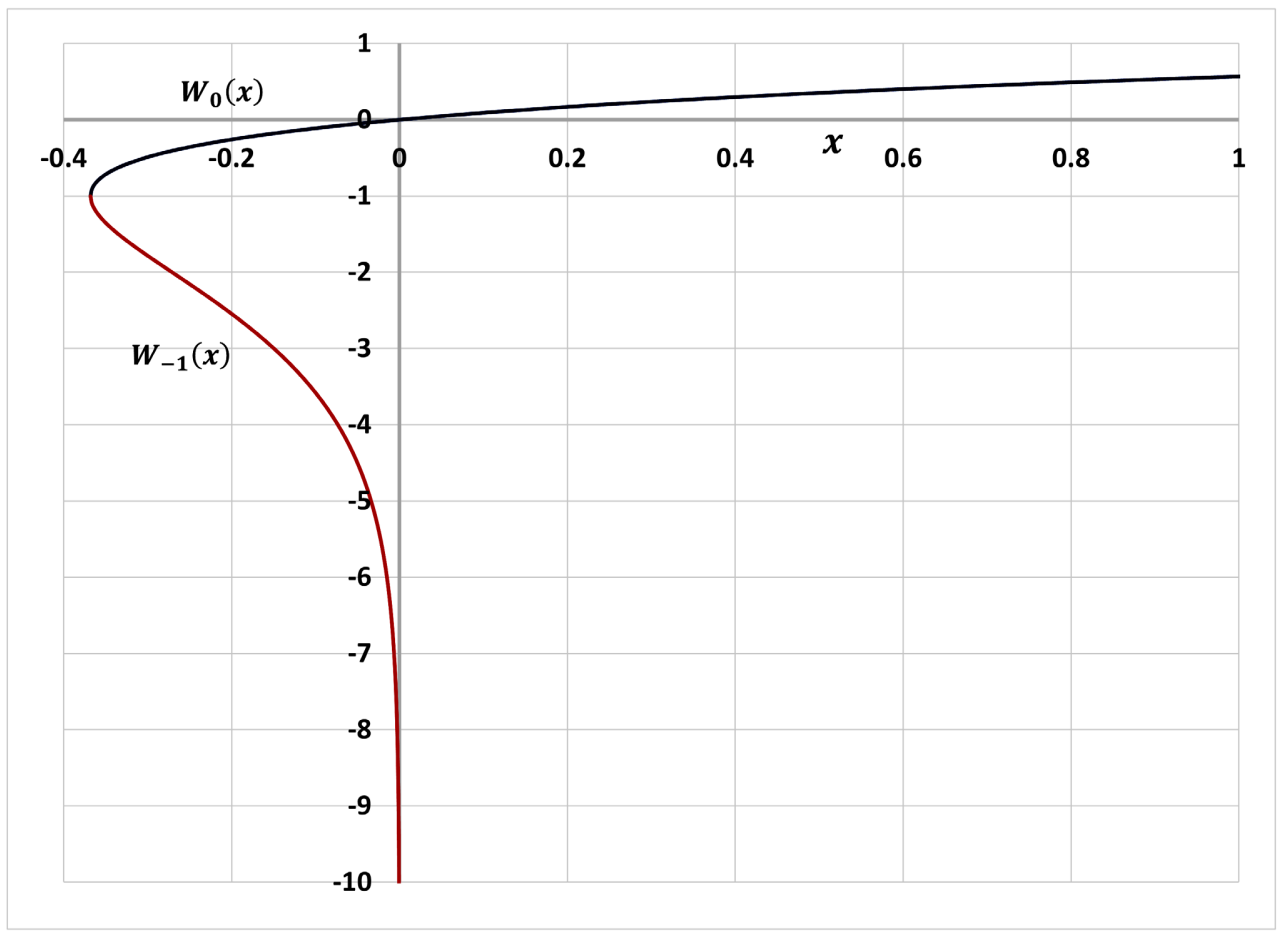

Figure 1. $W(\mathcal{X})$ vs $\mathcal{X}$ plot. 


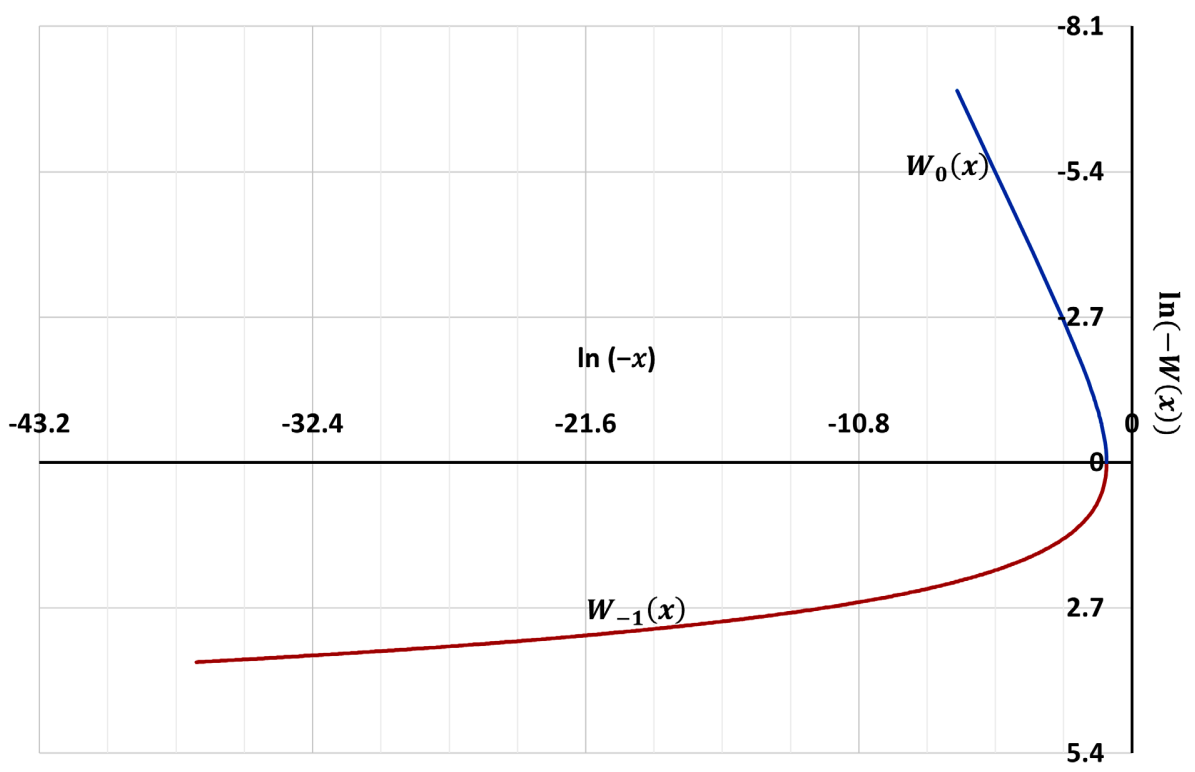

Figure 2. $\ln (-W(\mathcal{X}))$ vs $\ln (-\mathcal{X})$ plot.

This implies

$$
\begin{gathered}
\frac{v_{2}^{3}}{\mathrm{e}^{\frac{h v_{2}}{k T}}-1}=\frac{v_{1}^{3}}{\mathrm{e}^{\frac{h v_{1}}{k T}}-1} \\
\left(\frac{v_{1}}{v_{2}}\right)^{3} \mathrm{e}^{\frac{h v_{2}}{k T}}-\left(\frac{v_{1}}{v_{2}}\right)^{3}=\mathrm{e}^{\frac{h v_{1}}{k T}}-1 \\
\text { If } v_{2} \gg v_{1},\left(\frac{v_{1}}{v_{2}}\right)^{3} \ll 1 .
\end{gathered}
$$

The Equation (2.4) can be written as

$$
\left(\frac{v_{1}}{v_{2}}\right)^{3} \mathrm{e}^{\frac{h v_{2}}{k T}} \simeq \mathrm{e}^{\frac{h v_{1}}{k T}}-1
$$

For $\frac{h v_{1}}{k T} \ll 1$, region where Raleigh-Jean's law applies, the Equation (2.5) can be written as

$$
\left(\frac{v_{1}}{v_{2}}\right)^{3} \mathrm{e}^{\frac{h v_{2}}{k T}} \simeq \frac{h v_{1}}{k T}
$$

Let the ratio $\frac{v_{2}}{v_{1}}=r$.

The Equation (2.6) can be written as

$$
\frac{h v_{2}}{k T} \simeq \frac{1}{r^{2}} \mathrm{e}^{\frac{h v_{2}}{k T}}
$$




$$
\begin{gathered}
-\left(\frac{h v_{2}}{k T}\right) \mathrm{e}^{-\left(\frac{h v_{2}}{k T}\right)} \simeq-\left(\frac{1}{r^{2}}\right) \\
W\left(-\frac{1}{r^{2}}\right) \simeq-\frac{h v_{2}}{k T} \\
v_{2} \simeq \frac{k T}{h} W\left(-\frac{1}{r^{2}}\right) \text { and } v_{1}=\frac{v_{2}}{r}
\end{gathered}
$$

This new Equation (2.10) provides the solutions for the frequencies at which the intensities are equal, with the conditions $r \gg 1$ and $\frac{h v_{1}}{k T} \ll 1$. This equation is in the same form as the Equation (2.2) for the $v_{\max }$.

Table 1 gives the calculated values for the intensity ratio for the frequencies $v_{1}$ and $v_{2}$. The ratio is close to one for $r \gg 1$, as expected.

\section{Euler's Transcendental Equation and Lambert $W$ Function}

The solution for the equation $\mathcal{X}^{\mathcal{Y}}=\mathcal{Y}^{\mathcal{X}}$ is given by $\mathcal{Y}=\frac{W\left(-\frac{\ln (\mathcal{X})}{\mathcal{X}}\right)}{-\frac{\ln (\mathcal{X})}{\mathcal{X}}} \mathrm{de}$ rived by Euler in the $18^{\text {th }}$ century [1] [10] [11].

Theorem: The solutions for the series of exponential equations $\mathcal{Y}=\mathcal{X}^{\left(\frac{\mathcal{Y}}{\mathcal{X}^{n+1}}+n\right)}$ is given by $\mathcal{Y}=\mathcal{X}^{n} \exp \left[-W\left(-\frac{\ln (\mathcal{X})}{\mathcal{X}}\right)\right]$.

\section{Proof:}

One form of analytical solutions for the series of exponential equations was derived previously [10] [11].

$$
\mathcal{Y}=\mathcal{X}^{\left(\frac{\mathcal{Y}}{\mathcal{X}^{n+1}+n}\right)}
$$

The solutions derived previously:

$$
\mathcal{Y}=\mathcal{X}^{n+1}, \text { trivial solutions }
$$

and

Table 1. Intensity ratio for different $r$ values with other functions.

\begin{tabular}{ccccccc}
\hline$r$ & $-\frac{1}{r^{2}}$ & $-W_{-1}\left(-\frac{1}{r^{2}}\right)=\frac{h v_{2}}{k T}$ & $\frac{h v_{1}}{k T}$ & $\frac{v_{2}^{3}}{\mathrm{e}^{\frac{h v_{2}}{k T}}-1}$ & $\frac{v_{1}^{3}}{\mathrm{e}^{\frac{h v_{1}}{\mathrm{k} T}}-1}$ & $\begin{array}{c}\text { Intensity } \\
\text { ratio }\end{array}$ \\
\hline $1.65 \mathrm{E}+00$ & $-3.68 \mathrm{E}-01$ & 1 & $6.07 \mathrm{E}-01$ & $5.82 \mathrm{E}-01$ & $2.68 \mathrm{E}-01$ & 0.4597 \\
$5.45 \mathrm{E}+00$ & $-3.37 \mathrm{E}-02$ & 5 & $9.18 \mathrm{E}-01$ & $8.48 \mathrm{E}-01$ & $5.14 \mathrm{E}-01$ & 0.6062 \\
$4.69 \mathrm{E}+01$ & $-4.54 \mathrm{E}-04$ & 10 & $2.13 \mathrm{E}-01$ & $4.54 \mathrm{E}-02$ & $4.07 \mathrm{E}-02$ & 0.8972 \\
$4.67 \mathrm{E}+02$ & $-4.59 \mathrm{E}-06$ & 15 & $3.21 \mathrm{E}-02$ & $1.03 \mathrm{E}-03$ & $1.02 \mathrm{E}-03$ & 0.9840 \\
$4.93 \mathrm{E}+03$ & $-4.12 \mathrm{E}-08$ & 20 & $4.06 \mathrm{E}-03$ & $1.65 \mathrm{E}-05$ & $1.65 \mathrm{E}-05$ & 0.9980 \\
$5.37 \mathrm{E}+04$ & $-3.47 \mathrm{E}-10$ & 25 & $4.66 \mathrm{E}-04$ & $2.17 \mathrm{E}-07$ & $2.17 \mathrm{E}-07$ & 0.9998 \\
\hline
\end{tabular}




$$
\mathcal{Y}=\frac{\mathcal{X}^{n} W\left(-\frac{\ln (\mathcal{X})}{\mathcal{X}}\right)}{\left(-\frac{\ln (\mathcal{X})}{\mathcal{X}}\right)}, \text { non-trivial solutions }
$$

The non-trivial solutions can be refined further.

The Equation (3.1) can also be written as

$$
\frac{\ln \mathcal{Y}}{\ln \mathcal{X}}=\frac{\mathcal{Y}}{\mathcal{X}^{n+1}}+n
$$

Rearranging the Equation (3.4)

$$
\frac{\ln \mathcal{X}}{\mathcal{X}^{n+1}}=\frac{\ln \mathcal{Y}}{\mathcal{Y}}-\frac{n \ln \mathcal{X}}{\mathcal{Y}}
$$

Using Equation (3.5), the solution in Equation (3.3) can be written as:

$$
\ln \mathcal{Y}-n \ln \mathcal{X}=W\left(-\frac{\ln (\mathcal{X})}{\mathcal{X}}\right)
$$

Rearranging the Equation (3.6)

$$
\ln \mathcal{Y}=\ln \mathcal{X}^{n}+\ln \exp \left[W\left(-\frac{\ln (\mathcal{X})}{\mathcal{X}}\right)\right]
$$

Hence the solution for the Equation (3.1) can be written as

$$
\mathcal{Y}=\mathcal{X}^{n} \exp \left[-W\left(-\frac{\ln (\mathcal{X})}{\mathcal{X}}\right)\right]
$$

If $n=0$, the Equation (3.1) becomes

$$
\mathcal{X}=\mathcal{Y}^{\frac{\mathcal{Y}}{\mathcal{X}}}
$$

i.e. $\mathcal{X}^{\mathcal{Y}}=\mathcal{Y}^{\mathcal{X}}$ (Euler's equation).

The solution is

$$
\mathcal{Y}=\exp \left[-W\left(-\frac{\ln (\mathcal{X})}{\mathcal{X}}\right)\right]
$$

The $\frac{\ln (\mathcal{X})}{\mathcal{X}}$ is maximum at $\mathcal{X}=\mathrm{e}$. For $1<\mathcal{X}<\mathrm{e}$, the non-trivial solutions are in terms of $W_{0}(\mathcal{X})$ and for $\mathcal{X}>\mathrm{e}$, the non-trivial solutions are in terms of $W_{-1}(\mathcal{X})$.

\section{Numerical Calculation}

The numerical values of the function in Equation (3.10) were calculated using the Equation (3.9), utilizing the iterative technique. The iteration progresses are shown in Figure 3 for few $\mathcal{X}$ values. For $\mathcal{X}>\mathrm{e}$, the iteration converges to the non-trivial solution. For $\mathcal{X}<\mathrm{e}$, the iteration converges to the trivial solution. At $\mathcal{X}=\mathrm{e}, \mathcal{Y}=\mathrm{e}$, the trivial and the nontrivial solutions are equal.

The non-trivial solutions in the range of $1<\mathcal{X}<\mathrm{e}$ were determined, using the $\mathcal{X Y}$ symmetry in Equation (3.9). For $\mathcal{X}<\mathrm{e}$, even when the seed value 
close to the non-trivial solution the iteration is unstable (Figure 4).

The numerical values of the function $\exp \left[-W\left(-\frac{\ln (\mathcal{X})}{\mathcal{X}}\right)\right]$ are given in $\mathrm{Ta}$ ble 2 .

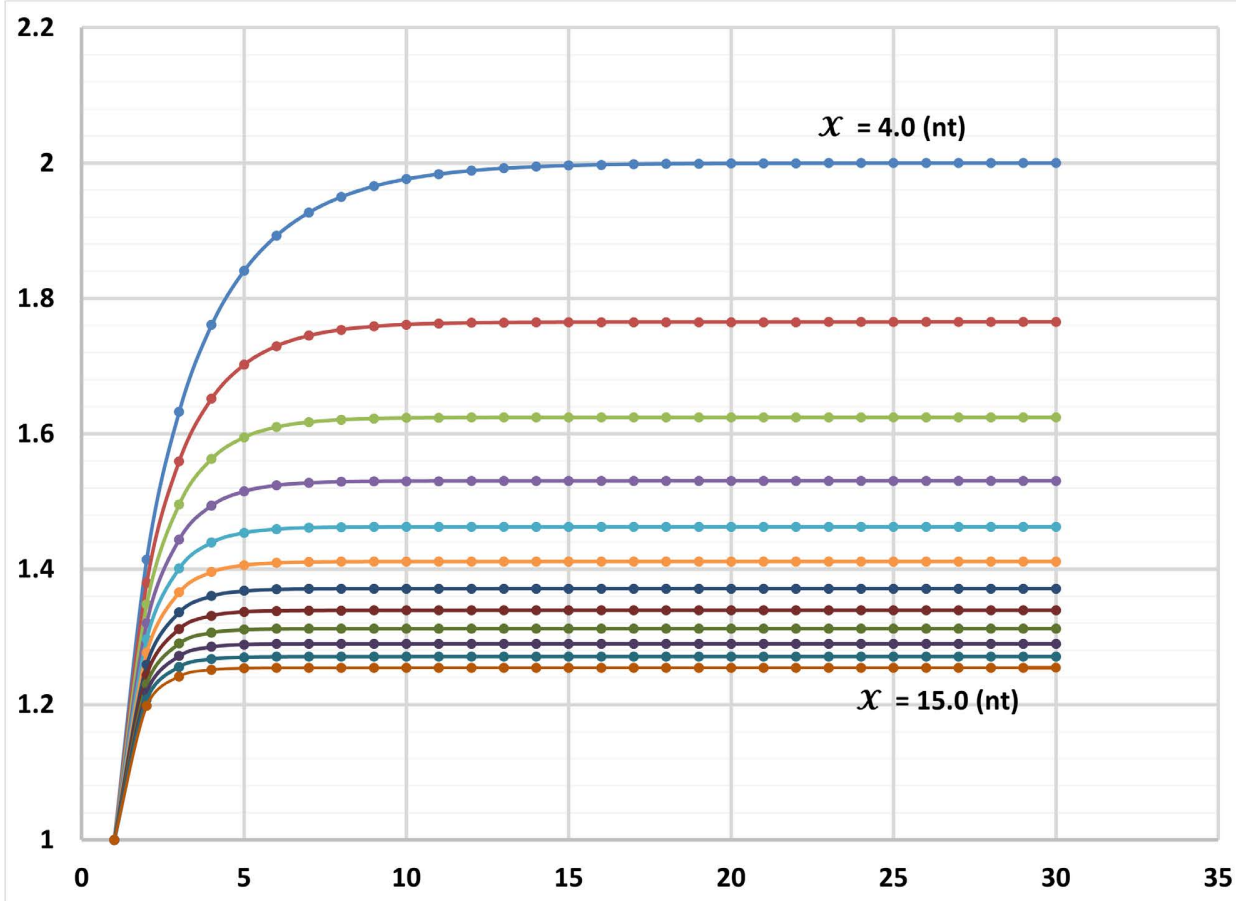

Figure 3. Iteration steps for non-trivial (nt) solutions for $\mathcal{X}$ values from 4 to 15.

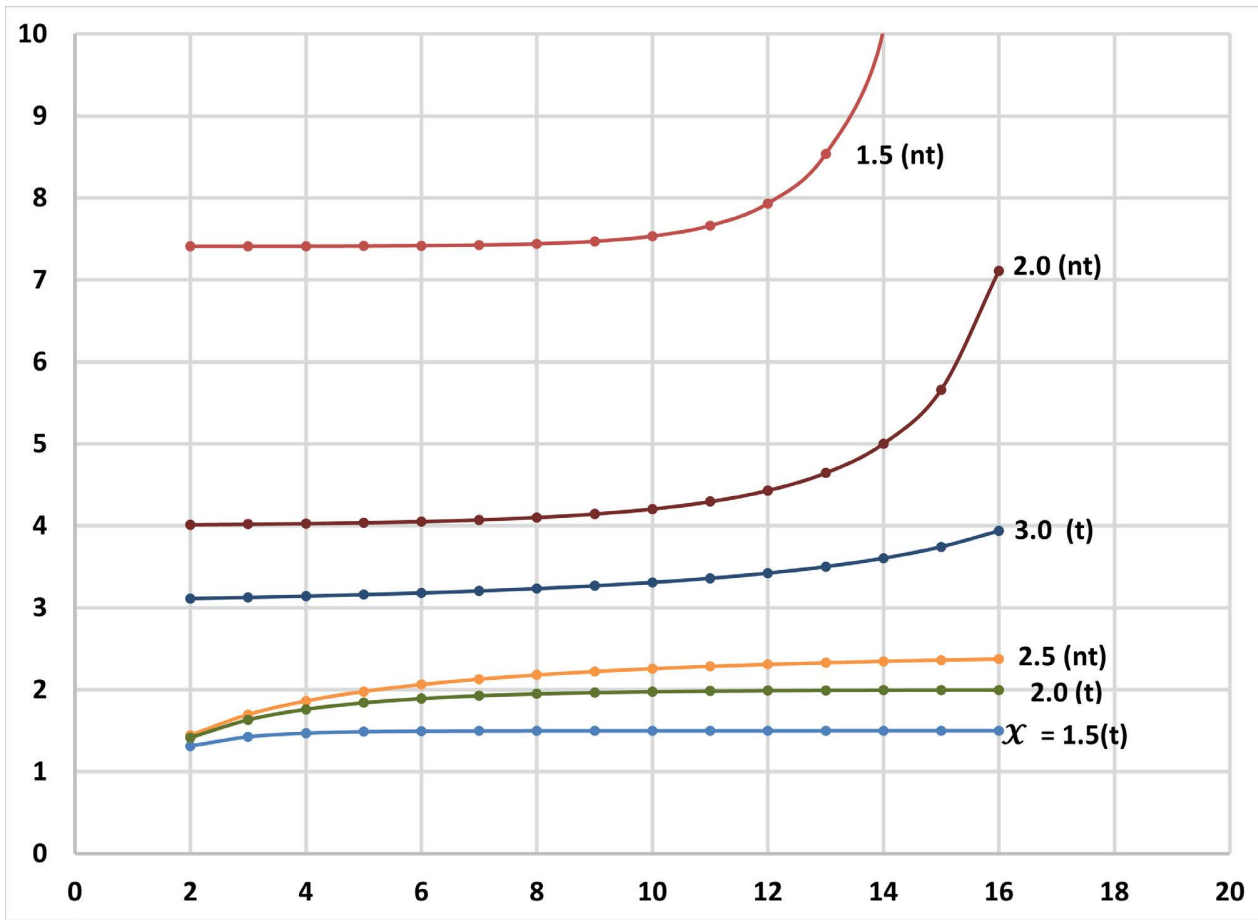

Figure 4. Iteration steps for trivial ( $\mathrm{t}$ ) and non-trivial (nt) solutions for $\mathcal{X}$ values of 1.5, 2 and 3. 
Table 2. Calculated values of function $\exp \left[-W\left(-\frac{\ln (\mathcal{X})}{\mathcal{X}}\right)\right]$, given in terms of $W_{0}$ and $W_{-1}$, depending on the range.

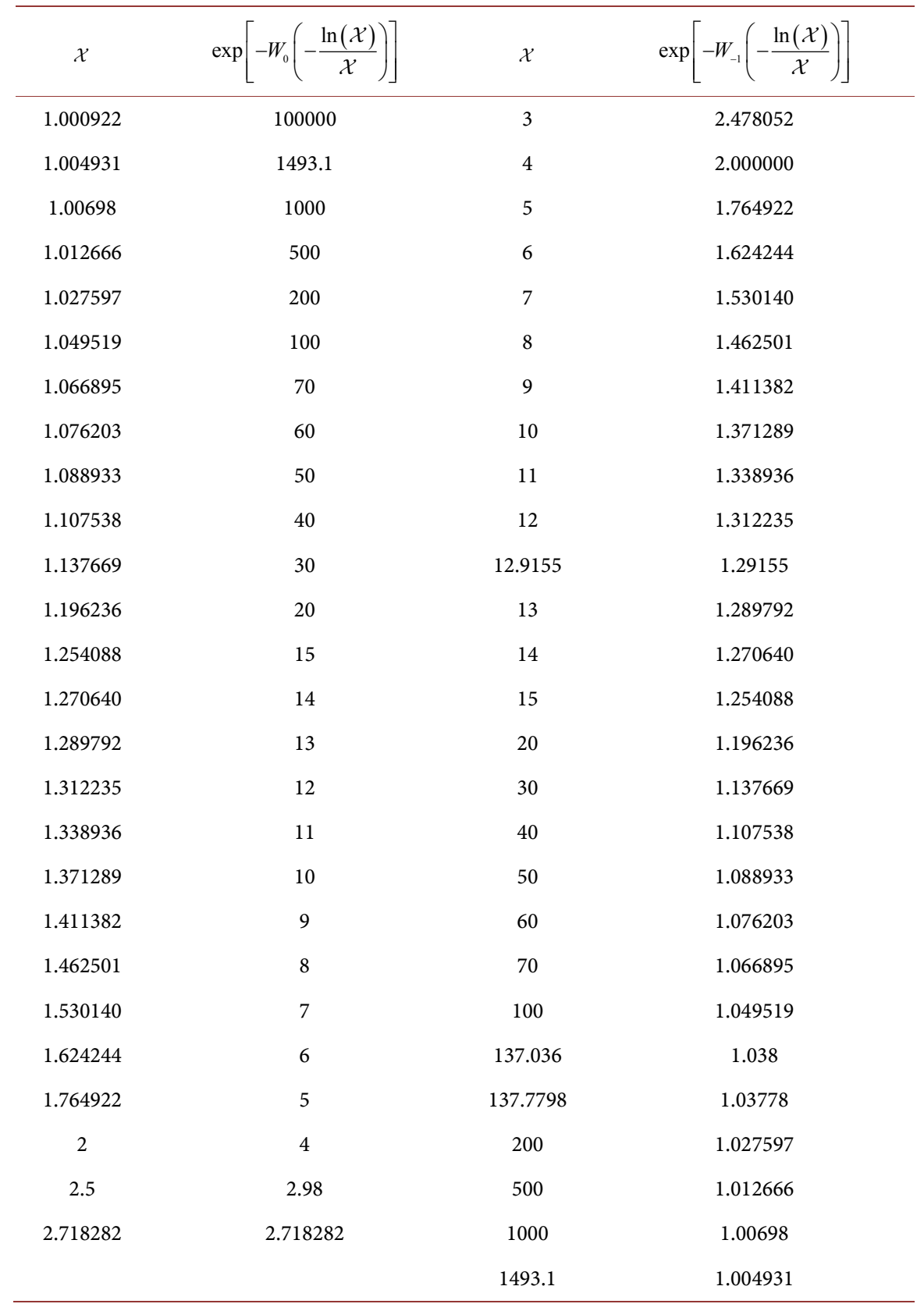

Using the values of the function in Table 2, the plots of the function $\mathcal{X}^{n} \exp \left[-W\left(-\frac{\ln (\mathcal{X})}{\mathcal{X}}\right)\right]$, for $n=-2,-1,0,1$ and 2 are shown in Figure 5. Plot of $-W\left(-\frac{\ln (\mathcal{X})}{\mathcal{X}}\right)$ calculated using the vales in Table 2 and the comparison plot of $\frac{\ln (\mathcal{X})}{\mathcal{X}}$ are shown in Figure 6. 


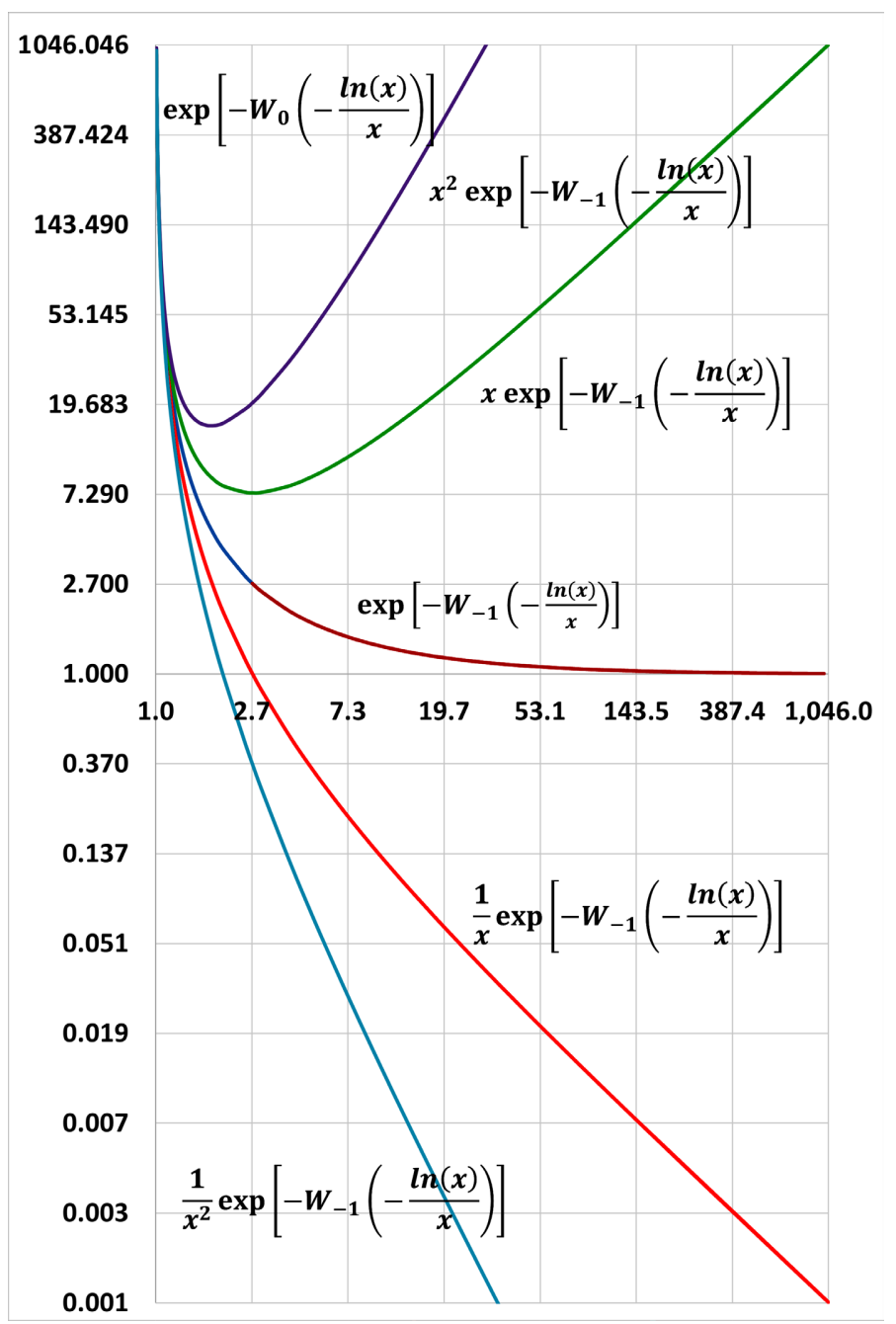

Figure 5. Plots of function $\mathcal{X}^{n} \exp \left[-W\left(-\frac{\ln (\mathcal{X})}{\mathcal{X}}\right)\right]$ for $\mathrm{n}$ of $-2,-1,0,1$, and 2 .

For different values of $n, \mathcal{X}$ and $\mathcal{Y}$ in Equation (3.8), using Table 2, following numerical equations can be obtained

$$
\begin{gathered}
2=\exp \left[-W_{-1}\left(-\frac{\ln (4)}{4}\right)\right] \\
4=\exp \left[-W_{0}\left(-\frac{\ln (2)}{2}\right)\right] \\
\exp \left[-W_{0}\left(-\frac{\ln (1.3713)}{1.3713}\right)\right]=10 \\
\exp \left[-W_{-1}\left(-\frac{\ln (10)}{10}\right)\right]=1.3713 \\
10^{1.3713}=1.3713^{10}=23.5
\end{gathered}
$$




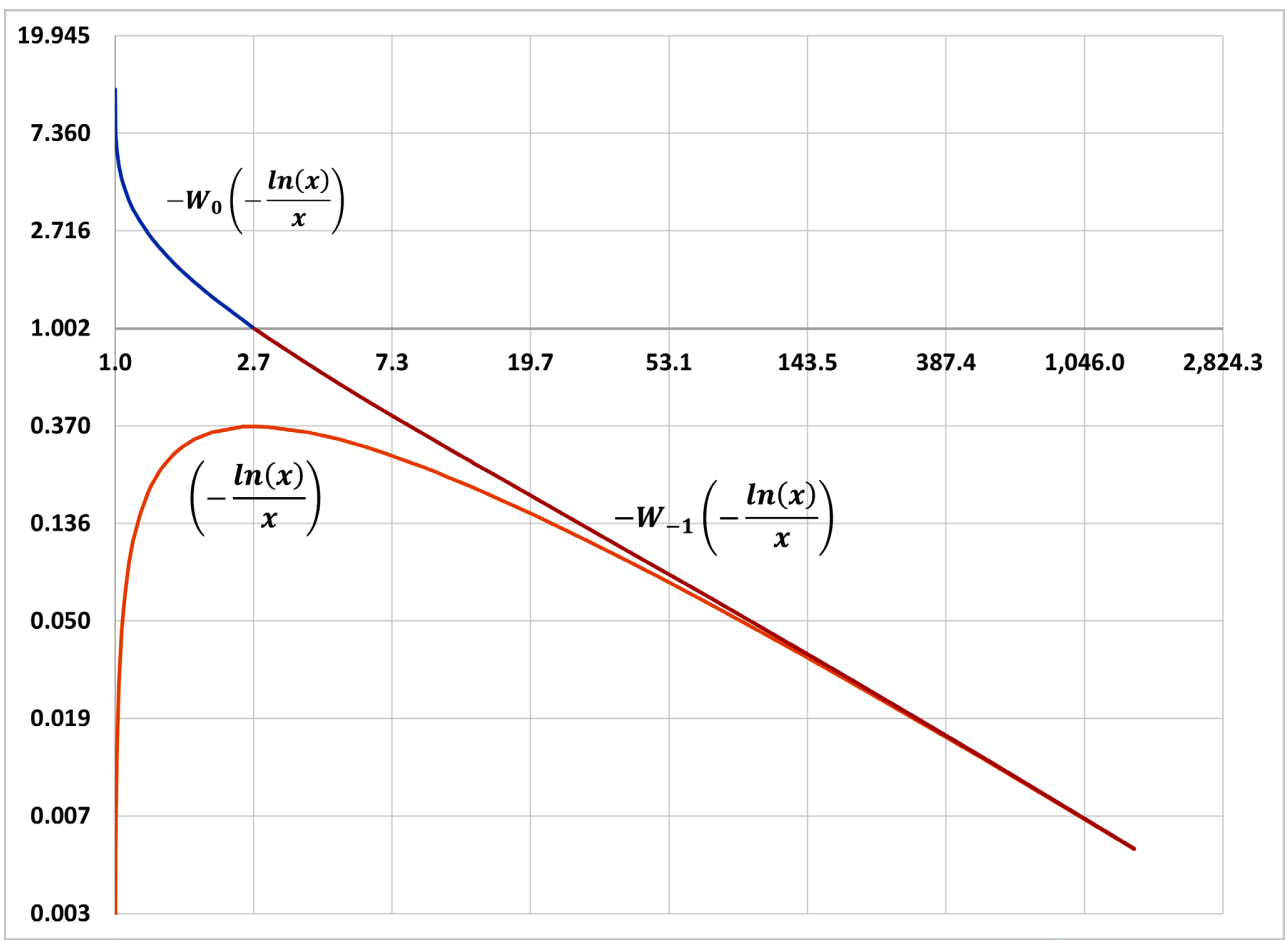

Figure 6. Plots of $-W\left(-\frac{\ln (\mathcal{X})}{\mathcal{X}}\right)$ and $\frac{\ln (\mathcal{X})}{\mathcal{X}}$.

$$
\begin{gathered}
\frac{\ln 1.3713}{1.3713}=\frac{\ln 10}{10}=0.2302 \\
10^{2} \exp \left[-W_{-1}\left(-\frac{\ln (10)}{10}\right)\right]=137.129 \\
\frac{\ln 137.13}{\ln 10}=\frac{137.13}{10^{3}}+2=2.13713 \\
\exp \left[-W_{-1}\left(-\frac{\ln (100)}{100}\right)\right]=1.0495 \\
\exp \left[-W_{-1}\left(-\frac{\ln (1000)}{1000}\right)\right]=1.00698 \\
1000^{1.00698}=1.00698^{1000} \\
12.9155^{1.29155}=1.2915^{12.9155} \\
137.78^{1.0378}=1.0378^{137.78} \\
1493.1^{1.004931}=1.004931^{1493.1}
\end{gathered}
$$

\section{Numerical Coincidences}

Consider the Equations (4.9) and (4.16), 


$$
\begin{aligned}
& 10^{2} \exp \left[-W_{-1}\left(-\frac{\ln (10)}{10}\right)\right]=137.129 \\
& \exp \left[-W_{0}\left(-\frac{\ln (1.0378)}{1.0378}\right)\right]=137.78
\end{aligned}
$$

These solutions are unique. Numerical coincidences for these numbers with physical constants are given below:

The dimensionless electromagnetic fine structure constant

$$
\begin{gathered}
\alpha=\frac{e^{2}}{4 \pi \varepsilon_{o} \hbar c}=7.2973 \times 10^{-3} \\
\alpha^{-1}=137.036 \\
\alpha^{-1}+10 \alpha=137.11 \\
\alpha^{-1}+100 \alpha=137.766 \\
\alpha^{-1}+\alpha^{1 / 2}+\alpha=137.1287
\end{gathered}
$$

The dimensionless gravitational fine structure constant defined using electron mass can be written as

$$
\alpha_{G}^{e e}=\frac{G m_{e}^{2}}{h c}=7.38 \times 10^{-45}
$$

For convenience hereafter $\alpha_{G}^{e e}$ will be referred as $\alpha_{G}$

$$
\begin{gathered}
\alpha_{G}^{-1}=1.35 \times 10^{44} \\
\ln \alpha_{G}^{-1}=101.6 \\
\left(\ln \alpha_{G}^{-1}\right)^{1 / 2}=10.08
\end{gathered}
$$

The numerical values of $\alpha^{-1}$ and $\ln \alpha_{G}^{-1}$ are close and it was suggested that they are related [12].

In Equation (4.9), if 10 is replaced with $\left(\ln \alpha_{G}^{-1}\right)^{1 / 2}$, the equation becomes

$$
\left(\ln \alpha_{G}^{-1}\right) \exp \left[-W_{-1}\left(-\frac{\ln \left(\left(\ln \alpha_{G}^{-1}\right)^{1 / 2}\right)}{\left(\ln \alpha_{G}^{-1}\right)^{1 / 2}}\right)\right]=136.85
$$

\section{Conclusion}

In the Planck's radiation law equation, for a specific temperature and intensity, the frequencies will be given by $v_{2} \simeq \frac{k T}{h} W\left(-\frac{1}{r^{2}}\right)$ and $v_{1}=\frac{v_{2}}{r}$, with conditions $r \ll 1$, and $\frac{h v_{1}}{k T} \ll 1$. The numerical calculations of the intensity at these frequencies validated the equations.

A new form of solution for the Euler's equation $\mathcal{X}^{\mathcal{Y}}=\mathcal{Y}^{\mathcal{X}}$ was derived in the form of the Lambert $W$ function as, $\mathcal{Y}=\exp \left[-W\left(-\frac{\ln (\mathcal{X})}{\mathcal{X}}\right)\right]$, and the corre- 
sponding solutions for the series of exponential equations. Interesting numerical equations were derived and coincidences with electromagnetic fine structure constant were indicated.

"God used beautiful mathematics in creating the world" quote by Paul Dirac.

\section{Conflicts of Interest}

The author declares no conflicts of interest regarding the publication of this paper.

\section{References}

[1] Corless, R.M., Gonnet, G.H., Hare, D.E.G., Jeffrey, D.J. and Knuth, D.E. (1996) On the Lambert $W$ Function. Advances in Computational Mathematics, 5, 329-359. https://doi.org/10.1007/BF02124750

[2] Mezo, I. and Keady, G. (2016) Some Physical Applications of Generalized Lambert Functions. European Journal of Physics, 37, Article ID: 065802. https://doi.org/10.1088/0143-0807/37/6/065802

[3] Mező, I. and Baricz, Á. (2017) On the Generalization of the Lambert $W$ Function. Transactions of the American Mathematical Society, 369, 7917-7934. https://doi.org/10.1090/tran/6911

[4] Belkić, D. (2018) The Euler Tand Lambert $W$ Functions in Mechanistic Radiobiological Models with Chemical Kinetics for Repair of Irradiated Cells. Journal of Mathematical Chemistry, 56, 2133-2193. https://doi.org/10.1007/s10910-018-0932-3

[5] Veberič, D. (2012) Lambert W Function for Applications in Physics. Computer Physics Communications, 183, 2622-2628. https://doi.org/10.1016/j.cpc.2012.07.008

[6] Valluri, S.R., Corless, R.M. and Jeffrey, D.J. (2000) Some Applications of the Lambert $W$ Function to Physics. Canadian Journal of Physics, 78, 823. https://doi.org/10.1139/p00-065

[7] Planck, M. (1901) On the Law of the Energy Distribution in the Normal Spectrum. Annalen der Physik, 4, 553.

[8] Valluri, S.R., Gil, M., Jeffrey, D.J. and Basu, S. (2009) The Lambert W Function in Quantum Statistics. Journal of Mathematical Physics, 50, Article ID: 102103. https://doi.org/10.1063/1.3230482

[9] Stewart, S.M. (2012) Spectral Peaks and Wien's Displacement Law. Journal of Thermophysics and Heat Transfer, 26, 689. https://doi.org/10.2514/1.T3789

[10] Gnanarajan, S. (2017) Solutions of the Exponential Equation $y^{x / y}=x$ or $\ln x / x=\ln y / y$ and Fine Structure Constant. Journal of Applied Mathematics and Physics, 5, 386-391. https://doi.org/10.4236/jamp.2017.52034

[11] Gnanarajan, S. (2018) Solutions for Series of Exponential Equations in Terms of Lambert-W Function and Fundamental Constants. Journal of Applied Mathematics and Physics, 6, 725-736. https://doi.org/10.4236/jamp.2018.64065

[12] Dirac, P.A.M. (1937) The Cosmological Constants. Nature, 139, 323. https://doi.org/10.1038/139323a0 